\title{
Highlights der Jahrestagung „Der herzkranke Diabetiker“
}

\section{METABOLISCH-VASKULÄRES SYNDROM}

\section{Eine neue Formel für komplexe Risiken}

Das Syndrom der vielen Namen: Vom tödlichen Quartett sprach man früher, dann vom Insulinresistenz-Syndrom, schließlich hieß es Metabolisches Syndrom, seit Kurzem auch Kardiometabolisches Syndrom und nun auch Metabolisch-Vaskuläres Syndrom, eine Wortschöpfung aus Sachsen. Die Fachkommission Diabetes Sachsen an der TU Dresden hat inzwischen eine Leitlinie vorgelegt und hofft, dass es in nicht allzu ferner Zukunft ein DMP Metabolisch-Vaskuläres Syndrom (MVS) gibt.

Das MVS gründet auf der „Common Soil“Hypothese. Danach sind viszerale Adipositas und genetische Disposition der Nährboden, auf dem sich metabolische und vaskuläre Störungen entwickeln bis hin zu Typ-2-Diabetes und kardiovaskulären Erkrankungen (s. Abb. 1).

Die Leitlinie ist eine komplexe Handlungsanleitung für Hausärzte und Diabe-

\section{NACHBETRACHTUNG DER STENO-STUDIE \\ Intensivierte Therapie senkt die Sterblichkeit}

Kaum ein Diabetesvortrag, in dem nicht auf die Steno-2-Studie als Beweis für die Erfolge einer intensivierten, multifaktoriellen Therapie des Typ-2-Diabetes verwiesen wird. Kürzlich ist eine Nachbetrachtung dieser richtungsweisenden Arbeit erschienen, die Prof. Petra-Maria SchummDraeger, München, vorstellte. Daraus lässt sich folgende Prognose ableiten: Von zehn Typ-2-Diabetikern mit Mikroalbuminurie im Alter von 55 Jahren werden in den nächsten 13 Jahren

- zwei sterben,

- zwei einen Schlaganfall bekommen,

- zwei einen Herzinfarkt erleiden,

- einer eine Amputation durchstehen, wenn nicht frühzeitig mit einer intensivierten, zielorientierten und strikt leitliniengemäßen Therapie begonnen wird. Wer dagegen eine solche intensivierte Therapie erhält, hat ein deutlich geringeres Mortalitätsrisiko: Gesamtsterblichkeit
Abbildung 1

Entstehung des MetabolischVaskulären Syndroms (MVS)

Rauchen u.a. exogene Risikofaktoren

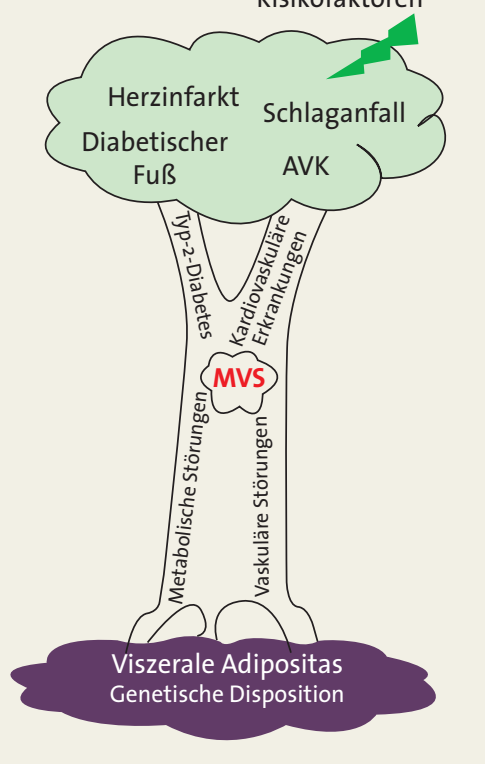

tologen und abrufbar unter der Fax-Nummer: 03 51/3 177233 .

\section{BEIM KARDIOLOGEN}

\section{Der Nichtdiabetiker ist die Ausnahme}

$75 \%$ aller Herzpatienten, darauf verwies Dr. Rolf Dörr, Dresden, haben einen manifesten Diabetes oder einen Prädiabetes. In einer Herzklinik oder Herzpraxis ist der Nichtdiabetiker der Ausnahmefall. Kardiologen sollten deshalb bei ihren KHK-Patienten gezielt nach einem Diabetes und Diabetologen bei ihren Diabetespatienten gezielt nach einer KHK suchen. Praktisch wichtig: Der Diabetiker ist ein kardialer Hochrisikopatient, bei dem die optimale Einstellung von Blutdruck, Glukosespiegel, Blutfetten und eine Lebensstil-Modifikation mit Gewichtsreduktion, mehr Bewegung, gesunder Ernährung und strenger Nikotinabstinenz von entscheidender Bedeutung sind.

Die nicht invasive Diagnostik der KHK darf sich bei Diabetikern nicht nur auf symptomatische Patienten konzentrieren, sondern muss auch die asymptomatischen erfassen. und kardiovaskuläre Sterblichkeit nach einem Zeitraum von 13 Jahren liegen absolut um 20 bzw. $13 \%$ niedriger und damit nur halb so hoch wie unter einer konventionellen Therapie, so das aufsehenerregende Ergebnis der Steno-2-Studie.

\section{EBM-ALARM BEI NIEDERGELASSENEN DIABETOLOGEN}

\section{„Vielen droht das Aus!“}

Der geplante neue EBM ist für viele niedergelassene Diabetologen existenzgefährdend. So sieht es zumindest Dr. Richard Daikeler vom Vorstand des Bundesverbandes niedergelassener Diabetologen, der auf die verheerenden Folgen für die Diabetikerbetreuung hinwies. Wird ein Hausarzt auf Überweisung durch einen anderen Hausarzt tätig, bekommt er nach dem neuen EBM für diesen Patienten nur noch eine halbierte Versichertenpauschale. Außerdem steht ihm die Chronikerpauschale nicht zu, erläuterte Daikeler. „Dies bedeutet, dass der haus- ärztlich tätige Diabetologe $30 \%$ seines Punktevolumens einbüßen wird, da keine Kompensationsmöglichkeit mehr besteht. Denn im neuen EBM werden Komplexleistungen geschaffen, die keine gesonderte Vergütung für Einzelleistungen wie Gespräch oder Schulung vorsehen. So ist mit einer Umsatzeinbuße von $30 \%$ zu rechnen - für viele existenzgefährdend.“

Auch für die niedergelassenen Diabetologen, die fachärztlich tätig sind, bringt der neue EBM eine böse Überraschung: Die „ausführliche Gesprächsziffer" 13220 soll abgeschafft werden. Damit entfällt eine adäquate Vergütung für das ausführliche Diabetesgespräch. „Das bedeutet, dass die primäre Tätigkeit des Diabetologen, Patienten zu beraten und im Rahmen der Therapieführung zu betreuen, nicht mehr wirtschaftlich abrechenbar sein wird ohne zusätzlich zu erbringende technische Leistung - die nicht immer erforderlich ist", so Daikeler.

DR. MED. JOCHEN AUMILLER -

- Quelle: 7. Jahrestagung „Der herzkranke Diabetiker", Berlin, 7./8. Dezember 2007 